\title{
Anti-malaria humoral responses in children exposed to Plasmodium falciparum and Schistosoma haematobium
}

\author{
F Mutapi $/{ }^{+}$, C Roussilhon ${ }^{*}$, T Mduluza ${ }^{* *}$, P Druilhe*
}

Institute for Immunology and Infection Research, School of Biological Sciences, Ashworth Laboratories, King's Buildings, University of Edinburgh, West Mains Road, Edinburgh EH9 3JT, UK * Bio-medical Parasitology, Institut Pasteur, Paris, France ${ }^{* *}$ Department of Biochemistry, University of Zimbabwe, Mount Pleasant, Harare

Antibody responses directed against the Plasmodium falciparum antigens, total extract, anti-merozoite surface protein-3 (MSP3b) and glutamate-rich protein (Glurp-RO) were studied in 42 children exposed to both Schistosoma haematobium and $\mathrm{P}$. falciparum infections. The association between levels of the anti-malaria IgG subclasses and IgM with host age, sex, schistosome infection intensity and schistosome specific antibodies was studied before chemotherapeutic treatment of schistosome infections. This showed a significant negative association between schistosome infection intensity and levels of IgG1, IgG3, and IgG4 directed against malaria total extract antigen, and a positive association between levels of anti-schistosome soluble egg antigen IgG2, IgG3, and IgG4 and levels of the same subclasses directed against malaria total extract antigens. The effect of treating schistosome infections with praziquantel on malaria specific responses was also studied. This treatment resulted in increases in significant IgG4 levels against MSP3b and IgM against Glurp RO. Treatment also resulted in a significant decrease in IgG4 levels against Glurp RO. Host age, sex or pre-treatment infection intensity was not associated with the magnitude of change in the two IgG4 responses while males showed a significantly higher increase in levels of IgM. The results suggest cross reactivity between schistosome and malaria antigens in this population.

Key words: Plasmodium falciparum - Schistosoma haematobium - co-infection - antibody - Zimbabwe

The geographical and socio-economic distribution of malaria infection overlaps with that of many helminth infections. This gives potential for interaction in the overall susceptibility, pathology or clinical manifestations of the infections. Experimental work has shown that schistosome and malaria infections profoundly affect each other, both at an immunological level and degree of pathology. For example, Plasmodium chabaudi parasitemia was significantly higher in Schistosoma mansoni infected mice, and T-cell responses are significantly reduced in affected mice (Helmby \& TroyeBlomberg 1998). There are numerous experiments showing a strong influence on parasite loads, course of infection, and outcome in animals concomitantly infected by two different protozoa species (Cox 2001). Three independent field studies in humans have shown that children carrying either intestinal helminth infections or $S$. haematobium are more susceptible to acute malaria attacks (Jambou et al. 1998, Spiegel et al. 2003, Sokhna et al. 2004). Interactions between different pathogen species have also been demonstrated by work on mycobacterial infection and helminths showing that BCG vaccinated people are less susceptible to intestinal nematodes, while pre-natal sensitisation to schistosome and/

Financial support: Birkbeck College, London, the Medical Research Council

+Corresponding author: f.mutapi@ed.ac.uk

Received 9 March 2007

Accepted 24 April 2007 or filarial antigens in Kenyan children reduced the efficacy of the BCG vaccine perhaps by biasing the immune response to produce IL-4 and IL-5 (Malhotra et al. 1999). A study previously conducted on this Zimbabwean population demonstrated that children coinfected with $S$. haematobium and $P$. falciparum produced significantly larger amounts of anti-schistosome egg $\mathrm{IgE}$ and $\mathrm{IgG} 3$ when compared to children coinfected with schistosomes alone (Mutapi et al. 2000). Recently work conducted on the effects of intestinal helminth infections on falciparum malaria in Thailand has produced conflicting results, suggesting that while Ascaris lumbricoides infection may protect against cerebral malaria (Nacher et al. 2002), intestinal helminths including A. lumbricoides increase the incidence of malaria attacks (Nacher et al. 2002). The authors suggest that a helminthmediated Th2 shift may decrease antisporozoite immunity, but protect against severe malaria (Nacher et al. 2002) as has also been more recently suggested for $S$. haematobium infection (Lyke et al. 2005). This study investigates the effect of schistosome infection intensity and schistosomespecific isotype levels on the level of humoral responses to specific malaria antigens. In addition, the effect of treating the schistosome infection with an antihelminth drug on the malaria responses is investigated. We focus on children aged between 6 and 14 years old as this is where the most dramatic changes in schistosome infection levels occur in this population (Mutapi et al. 1997).

\section{MATERIALS AND METHODS}

Study population - The children who took part in this study were part of a larger study investigating the effect of schistosome infection history on the development of antischistosome immune responses as previously de- 
scribed (Hagan et al. 1994, Mutapi et al. 1997). The children came from compliant participants (or permission granted by parents) whose families were permanent residents of the study area. The selection of the study area, and study population are described in detail elsewhere (Mutapi et al. 1997). Briefly, the Burma Valley (eastern highlands of Zimbabwe), where there are a number of large farms with communities of farm workers was selected for the study after an initial survey of several $S$. haematobium endemic areas. P. falciparum, the predominant species of malaria in Zimbabwe occurs in this region. Malaria transmission here is seasonal occurring during October to March (Taylor Mutambu 1986). The study samples were collected before the start of the transmission period in August (pre-treatment of schistosome infection samples) and at the end of transmission in March (6 months after schistosome treatment samples) to encompass a single malaria transmission period.

Sample collection - At the start of the study, at least two urine samples and a single stool sample were collected from each child for S. haematobium and intestinal helminth detection. Stool samples were processed following the Kato Katz procedure (Katz et al. 1972) to detect S. mansoni eggs and other intestinal helminths while the urine filtration method (Mott 1983) was used to detect $S$. haematobium eggs in urine samples. $10 \mathrm{ml}$ of venous blood were collected from each participant for serological assays and a thin smear slide prepared for microscopic detection of $P$. falciparum, in August before the peak malaria transmission period. All children were exposed to P. falciparum infection, as they were permanent residents of the area, which is endemic for malaria. Following this, all participants were treated for schistosome infections with $40 \mathrm{mg}$ of praziquantel per $\mathrm{kg} / \mathrm{body}$ weight and the efficacy of the treatment checked six weeks later in September. All children were re-examined six months later in March for malaria and helminth infection (S. haematobium and intestinal parasites) and a blood sample also collected as before. To be included in the study cohort, participants had to meet all of the following criteria (1) provided at least two urine and two stool samples on consecutive days at all time points for schistosome and intestinal helminth parasite diagnosis; (2) been negative for intestinal helminths including $S$. mansoni at all time points; (3) been confirmed egg negative for $S$. haematobium infection at the second time point if they had been treated, and (4) given a blood sample at both time points (for malaria diagnosis and serological assays) A total of 42 children (16 female and 26 male) aged between 6 and 14 years, met these criteria and therefore formed the study cohort.

Immunological assays - Serological assays were carried out to determine the level of $\operatorname{IgM}, \operatorname{IgG} 1, \operatorname{IgG} 2, \mathrm{IgG} 3$, and IgG4 antibodies against schistosome soluble egg antigen (SEA) and three distinct malaria antigens (a total schizont extract, the MSP3b peptide and the Glurp R0 polypeptide). Indirect enzyme linked immunosorbent assays (ELISA) already published for S. haematobium (Mutapi et al. 1997) and for the malarial antigens (Oeuvray et al. 1994, Aribot et al. 1996, Theisen et al. 1998) were used for the assays.
Statistical analysis - For all statistical analyses, children were divided into two age groups, 6-10 years old and 11-14 years old. The effects of the categorical variables, age group and sex, and the continuous variables schistosome infection intensity [log $(x+1)$ transformed] and schistosome antibody level (square root transformed) on the level of malaria antibody responses were tested using analysis of variance. To determine if levels of malaria antibody responses changed significantly after treatment of schistosome infections, a repeated measures general linear model analysis was performed. This procedure also tested for the effects of sex, age group and pre-treatment schistosome infection intensity on the magnitude of change in antibody levels (transformed as before). The effect of the malaria status was also tested in the two analyses and found to be not significant. However, the variable was excluded from the analyses presented in the results section due to the small number of children positive for malaria $(\mathrm{n}=11)$ and those positive for both infections $(n=3)$. All statistical analyses were performed using the statistical software SPSS, and type III sums of squares were used to calculate the F-value and the p value was set at 0.05 (see Mutapi \& Roddam 2002). Factors whose p-values were greater or equal to this value following table-wide Bonferoni corrections were accepted as representing a significant effect (Rice 1989).

Ethics - Permission to conduct the study in the region was obtained from the Provincial Medical Director, while the study underwent ethical review by the Ministry of Health and Child Welfare in Zimbabwe and the internationally recognized Medical Research Council of Zimbabwe. Only compliant participants were recruited into the study and were free to drop out at any point during the study. At the beginning of the study, participants and their parents/guardians had the aims of the project explained fully and consent was sought from parents/ guardian before children are bled. Anti-helminth treatment was administered to all compliant people after the first survey as well as at the end of the study. In addition people presenting with malaria were treated according to the treatment regime prescribed by the Ministry of Health in Zimbabwe.

\section{RESULTS}

Before treatment of schistosome infections - The prevalence of $S$. haematobium was $42.8 \%$ while mean infection intensity was $13.9 \mathrm{eggs} / 10 \mathrm{ml}$ of urine (range $0-220$ ). Malaria infection prevalence was $26 \%$. Levels of malarial IgG1, IgG3, and IgG4 directed against total extract antigen were significantly associated with schistosome infection intensity when allowing for confounding variables. For all three isotypes, there was a negative association between schistosome infection intensity and the level of the isotype produced as shown in figure 1 for $\mathrm{IgG} 1$ and $\mathrm{IgG} 3$. Levels of $\mathrm{IgG} 2, \mathrm{IgG} 3$, and $\mathrm{IgG} 4 \mathrm{di}-$ rected against $P$. falciparum total extract antigen were significantly associated with levels of similar isotypes directed against $S$. haematobium soluble egg antigen after allowing for other confounding variables (Table). This association was positive. Sex significantly affected only levels of anti-MSP3b IgM (females producing less 
a

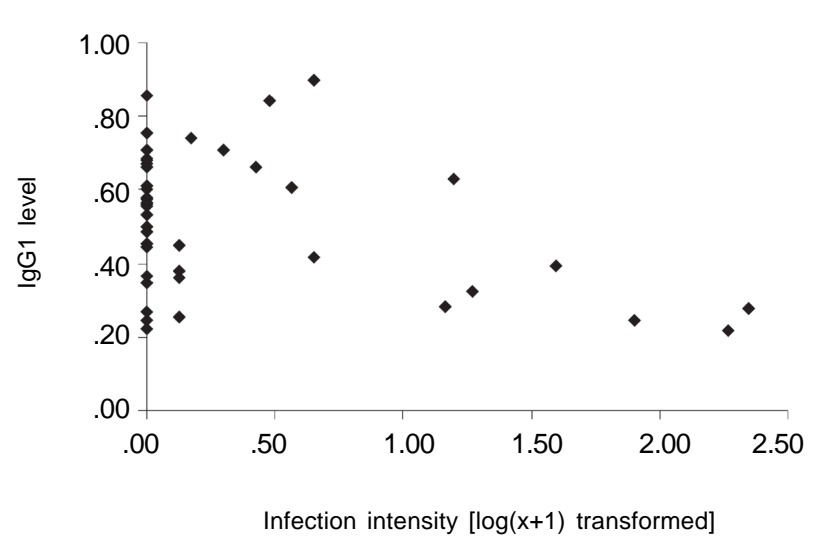

b

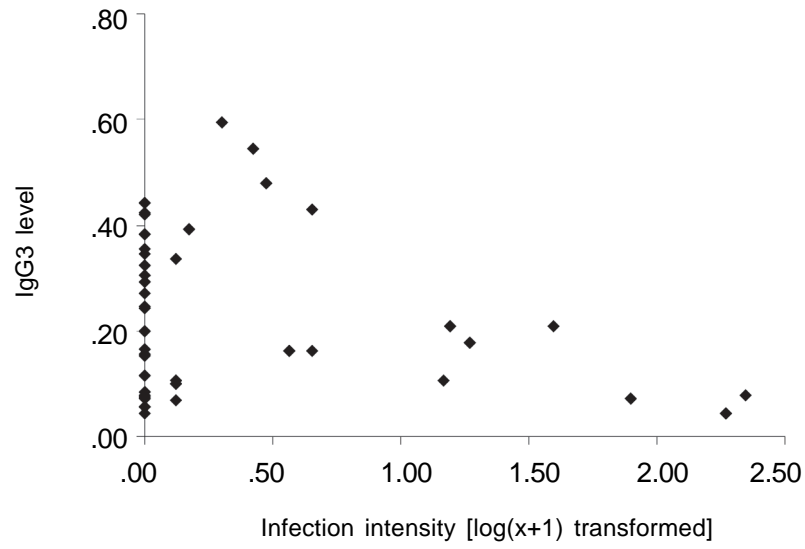

Fig. 1: negative correlation between anti-malaria antibody level and schistosome infection intensity. a: anti-malaria total extract IgG1; b: anti-malaria total extract $\operatorname{IgG} 3$.

TABLE

Statistical output for analysis of variance determining factors affecting the level of anti-malaria antibody responses before antihelminth treatment. The table gives F-values for each factors tested

\begin{tabular}{|c|c|c|c|c|}
\hline & Dependent variable & Total extract & MSP3B & Glurp R0 \\
\hline \multirow[t]{4}{*}{ IgG1 } & Age group & 0.6 & 0.8 & 0.5 \\
\hline & Sex & $7.7 * *$ & 0.01 & 0.04 \\
\hline & Pre-treatment schistosome infection intensity & $7.2 * *$ & 0.2 & 0.3 \\
\hline & Pre-treatment schistosome IgG1 & 1.4 & 1 & 0.3 \\
\hline \multirow[t]{4}{*}{$\operatorname{IgG} 2$} & Age group & 0.5 & 0.01 & 1.1 \\
\hline & Sex & 0.4 & 1.9 & 0.001 \\
\hline & Pre-treatment schistosome infection intensity & 0.5 & 0.4 & 0.7 \\
\hline & Pre-treatment schistosome IgG2 & $8.0 * *$ & 0.1 & $4.6^{*}$ \\
\hline \multirow[t]{4}{*}{ IgG3 } & Age group & 2.3 & 1.4 & 0.1 \\
\hline & Sex & 1.4 & 2.9 & 3.3 \\
\hline & Pre-treatment schistosome infection intensity & $8.7 * *$ & 1.8 & 1.7 \\
\hline & Pre-treatment schistosome IgG3 & $11.3^{* *}$ & 3.4 & 1.1 \\
\hline \multirow[t]{4}{*}{ IgG4 } & Age group & 0.0 & 1.1 & 0.7 \\
\hline & Sex & 1.0 & 2.2 & 1.2 \\
\hline & Pre-treatment schistosome infection intensity & $4.4^{*}$ & 2.2 & 4.1 \\
\hline & Pre-treatment schistosome IgG4 & $5.8^{*}$ & 0.5 & 1.8 \\
\hline \multirow[t]{4}{*}{$\operatorname{IgM}$} & Age group & 0.5 & 0.5 & 0.7 \\
\hline & Sex & 0.2 & $15.8^{* * *}$ & 4 \\
\hline & Pre-treatment schistosome infection intensity & 0.2 & 0.2 & 0.6 \\
\hline & Pre-treatment schistosome IgM & 0.1 & 1.5 & 0.2 \\
\hline
\end{tabular}

Degrees of freedom $=1$, for all variables and total degrees of freedom $=41, *=\mathrm{P}<=0.05, * * * \mathrm{P}<=0.01$ following Bonferoni correction.

than males), while age group did not have a significant effect on the levels of antibodies produced against any of the malaria antigens.

Six months after anti-helminth treatment - Three children were re-infected with schistosomiasis whereas there was no change in the prevalence of malaria with the same children who were positive for malaria infection before anti-helminth treatment still being positive for $P$. falciparum infection. There were also no changes in the levels of anti- total extract antigen but there were changes in responses directed against MSP3b and Glurp-
R0 antigens as shown in Fig. 2. There were significant increases in levels of $\operatorname{IgG} 4(\mathrm{~F}=12.9$, df $=27,1, \mathrm{p}=$ 0.002 ) directed against MSP3b (Fig. 2a) and IgM (F = 25.8, DF $=27,1, \mathrm{P}<0.001)$ directed against Glurp R0 (Fig. 2b). The magnitude of these changes was not related to host age, or pre-treatment infection intensity. However, the increase in IgM levels was significantly higher in males than females $(\mathrm{F}=4.8$, df $=1,27, \mathrm{p}=$ 0.037). Levels of IgG4 directed against Glurp R0 decreased significantly $(\mathrm{F}=21, \mathrm{DF}=27,1, \mathrm{P}<0.001)$ following schistosome treatment (Fig. 2b). 
a

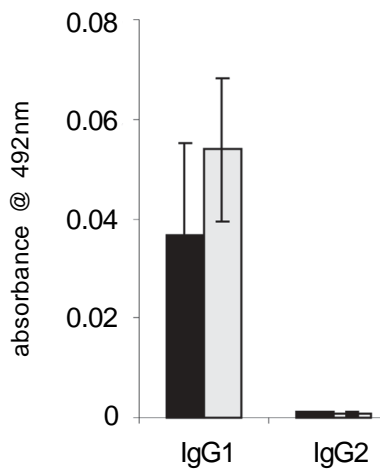

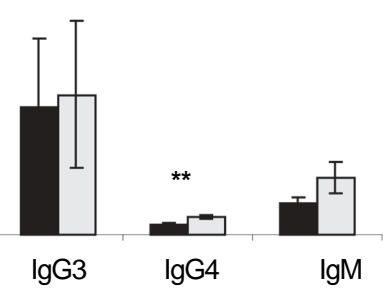

b

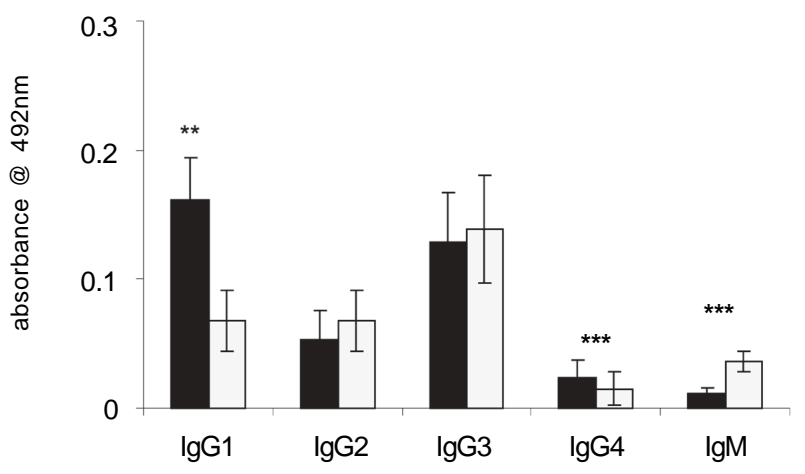

Fig. 2: antibody levels directed against malaria antigens before and 6 months after treatment of Schistosoma haematobium with praziquantel. Dark shaded bars represent pre-treatment antibody levels, light bars represent antibody levels 6 months post anti-helminth treatment; **represents a difference between pre and post treatment levels which is significant at $\mathrm{p}<0.01$, and $* * *$ at $\mathrm{p}<0.001$. a: antibody responses against MSP3b; b: antibody responses against GlurbR0.

\section{DISCUSSION}

The results from this study show two important aspects of the interaction between $S$. haematobium and $P$. falciparum infections. First, at low schistosome infection intensities, levels of the cytophilic isotypes $\operatorname{IgG} 1$, $\mathrm{IgG} 3$, and $\mathrm{IgG} 4$ directed against malaria total extract are high and they decline significantly with increasing schistosome infection intensity. In addition levels of IgG2, $\mathrm{IgG} 3$, and $\mathrm{IgG} 4$ directed against the same antigen were positively correlated with levels of the same isotypes directed against schistosome soluble egg antigen. This suggests that there may be cross-reactivity between the antigens from the two parasites, especially since the correlations are observed between responses to two crude antigens with the potential of having more cross reactive epitopes. Recently, work by Naus et al showed cross reactivity between IgG3 directed against $P$. falciparum MSP1 and S. mansoni adult worm antigens in people exposed to both parasites (Naus et al. 2003). They suggested that rather than having cross-reactive epitopes the two antigens might have molecules such as lecithins that are cross-reactive. In our study there was no association between schistosome infection intensity/ specific antibodies with responses directed against MSP3b. The only association with responses to Glurp R0 was a significant negative correlation between $\operatorname{IgG} 2$ directed against schistosome-SEA and Glurp R0. It remains to be established if the cross-reactivity with schistosome antigens does indeed offer some protection against malaria infections. Both IgG1 and IgG3 have been associated with acquired resistance to malaria infections (Bouharoun-Tayoun \& Druihle 1992, Aribot et al. 1996). An understanding of the immunological, pathological and epidemiological interaction of the two infections is important as indicated by field observations showing that people carrying helminth infections have increased frequency of acute malaria attacks (Jambou et al. 1998, Nacher et al. 2002, Spiegel et al. 2003, Sokhna et al. 2004, Lyke et al. 2006). Indeed, field studies in Madagascar and Senegal showed an increased number of malarial infections in people carrying high worm loads at- tacks (Jambou et al. 1998, Spiegel et al. 2003, Sokhna et al. 2004).

The conflicting reports of the effects of intestinal helminths on susceptibility to malaria infection (Nacher et al. 2000, 2002) suggest that the effect of helminth infections on susceptibility/resistance to malaria is a result of several as yet undefined factors. These will include the history of infection for infections (frequency, duration and order of establishment), helminth infection intensity (as indicated by our results), malaria parasitemia levels and background immunity to either pathogen. The effect of helminth infections on the susceptibility to malaria seems to depend on the malaria clinical state of the patient (Jambou et al. 1998, Nacher et al. 2000, 2002, Spiegel et al. 2003, Sokhna et al. 2004).

The second result in this study shows that 6 months after schistosome treatment, there were significant increases of IgG4 levels against MSP3b and IgM against Glurp R0 as well as a significant decrease in IgG4 levels against Glurp R0, but not the crude antigen, total extract. Host age, sex or pre-treatment infection intensity was not associated with the magnitude of change in the two IgG4 responses while males showed a significantly higher increase in levels of $\operatorname{IgM}$.

While these changes might be related to cross-reactive schistosome antigens released from dying worms (it is already know that treatment of schistosome infections results in exposure of schistosome antigens normally unavailable to the immune system (Mutapi et al. 2005), it is not clear that these changes are attributable to the effects of praziquantel treatment on schistosome worms. The changes may be reflecting the normal dynamics of anti-malaria responses over time, in this population. To explicitly test the effects of anti-helminth treatment on the malaria responses requires the use of untreated age, sex and malaria-exposure matched controls which were not included in this study for ethical reasons. There is also potential for influence of co-infections on the cytokine milieu for parasite specific responses, this too remains to be investigated. Immunological and pathological effects of treating human hel- 
minth infections on malaria remain unclear. Results from studies addressing this aspect could have profound implications for both malaria and helminth control programmes. Therefore detailed studies using larger sample sizes, across a wider age range and different epidemiological settings of the two infections are needed to further current understanding of the interaction of the two parasites.

\section{ACKNOWLEDGMENTS}

To the participants in the Burma Valley, technical staff at the Blair Research Institute and Michael Theisen for the gift of the purified Glurp-R0 recombinant antigen used in the study.

\section{REFERENCES}

Aribot G, Rogier C, Sarthou JL, Trape JF, Balde AT, Druilhe P, Roussilhon C 1996. Pattern of immunoglobulin isotype response to Plasmodium falciparum blood-stage antigens in individuals living in a holoendemic area of Senegal (Dielmo, West Africa). Am J Trop Med Hyg 54: 449-57.

Bouharoun-Tayoun H, Druihle P 1992. Plasmodium falciparum: evidence for an isotype imbalance which may be responsible for delayed acquisition of protective immunity. Infect Immun 60: 1473-1481.

Cox F 2001. Concomitant infections, parasites and immune responses. Parasitology 122 (Suppl.): S23-S38.

Hagan P, Chandiwana S, Ndhlovu P, Woolhouse M, Dessein A 1994. The epidemiology, immunology and morbidity of Schistosoma heamatobium infections in diverse communities in Zimbabwe. Trop Geogr Med 46: 227-232.

Helmby H, Troye-Blomberg M 1998. Altered immune repsonses in mice with concomitant Schistosoma mansoni and Plasmodium chabaudi infections. Infect Immunity 65: 3271-3276.

Jambou R, Rasamoel P, Ralamboranto L, Milijoana L, Raharimalala L, Pecarere JL, Druihle P 1998. Change in response to malaria induced by repeated treatment of children with Levamisole. Gordon Conferences, Oxford University, UK.

Katz N, Chaves A, Pellegrino J 1972. A simple device for quantitative stool thick smear technique in schistosomiasis mansoni. Rev Inst Med Trop São Paulo 14: 397-400.

Lyke KE, Dabo A, Sangare L, Arama C, Daou M, Diarra I, Plowe CV, Doumbo OK, Sztein MB 2006. Effects of concomitant Schistosoma haematobium infection on the serum cytokine levels elicited by acute Plasmodium falciparum malaria infection in Malian children. Infect Immun 74: 5718-24.

Lyke KE, Dicko A, Dabo A, Sangare L, Kone A, Coulibaly D, Guindo A, Traore K, Daou M, Diarra I, Sztein MB, Plowe CV, Doumbo OK 2005. Association of Schistosoma haematobium infection with protection against acute Plasmodium falciparum malaria in Malian children. Am J Trop Med Hyg 73: 1124-30.

Malhotra T, Mungai P, Wamachi A, Kioko J, Ouma JH, Kazura JW, King LK 1999. Helmnth and Bacillus Calmette-Guerin induced immunity in children sensitized in utero to filariasis and schistosomiasis. J Immunol. 162: 6843-6848.

Mott KE 1983. A reusable polyamide filter for diagnosis of $S$. haematobium infection by urine filtration. Bull Soc Pathol Exot 76: 101-104.

Mutapi F, Burchmore R, Foucher A, Harcus Y, Nicoll G, Midzi N,
Turner C, Maizels R 2005. Praziquantel treatment of people exposed to Schistosoma haematobium enhances serological recognition of defined parasite antigens. J Infect Dis 192: 1108-1118.

Mutapi F, Hagan P, Ndhlovu P, Woolhouse MEJ 1997. Comparison of humoral responses to Schistosoma haematobium in areas with high and low levels of infection. Parasite Immunol 19: 255-263.

Mutapi F, Ndhlovu PD, Hagan P, Woolhouse MEJ 2000. Antischistosome antibody responses in children coinfected with malaria. Parasite Immunol 22: 207-209.

Mutapi F, Roddam A 2002. p-values for pathogens: statistical inference from infectious-disease data. Lancet Infect Dis 2: 219-230.

Nacher M, Gay F, Singhasivanon P, Krudsood S, Treeprasertsuk S, Mazier D, Vouldoukis I, Looareesuwan S 2000. Ascaris lumbricoides infection is associated with protection from cerebral malaria. Parasite Immunol 22: 107-13.

Nacher M, Singhasivanon P, Treeprasertsuk S, Vannaphan S, Traore B, Looareesuwan S, Gay F 2002. Intestinal helminths and malnutrition are independently associated with protection from cerebral malaria in Thailand. Ann Trop Med Parasitol 96: 5-13.

Nacher M, Singhasivanon P, Yimsamran S, Manibunyong W, Thanyavanich N, Wuthisen R, Looareesuwan S 2002. Intestinal helminth infections are associated with increased incidence of Plasmodium falciparum malaria in Thailand. J Parasitol 88: 55-8.

Naus CW, Jones FM, Satti MZ, Joseph S, Riley EM, Kimani G, Mwatha JK, Kariuki CH, Ouma JH, Kabatereine NB, Vennervald BJ, Dunne DW 2003. Serological responses among individuals in areas where both schistosomiasis and malaria are endemic: cross-reactivity between Schistosoma mansoni and Plasmodium falciparum. J Infect Dis 187: 1272-82.

Oeuvray C, Bouharoun-Tayoun H, Gras-Masse H, Bottius E, Kaidoh T, Aikawa M, Filgueira MC, Tartar A, Druilhe P 1994. Merozoite surface protein-3: a malaria protein inducing antibodies that promote Plasmodium falciparum killing by cooperation with blood monocytes. Blood 84: 1594-602.

Rice WR 1989. Analysing tables of statistical tests. Evolution 43: 223-225.

Sokhna C, Le Hesran JY, Mbaye PA, Akiana J, Camara P, Diop M, Ly A, Druilhe P 2004. Increase of malaria attacks among children presenting concomitant infection by Schistosoma mansoni in Senegal. Malar J 3: 43.

Spiegel A, Tall A, Raphenon G, Trape JF, Druilhe P 2003. Increased frequency of malaria attacks in subjects co-infected by intestinal worms and Plasmodium falciparum malaria. Trans $R$ Soc Trop Med Hyg 97: 198-9.

Taylor Mutambu SL 1986. A review of the malaria situation in Zimbabwe with special reference to the period 1972-1981. Trans $R$ Soc Trop Med Hyg 80: 12-19.

Theisen M, Soe S, Oeuvray C, Thomas AW, Vuust J, Danielsen S, Jepsen S, Druilhe P 1998. The glutamate-rich protein (GLURP) of Plasmodium falciparum is a target for antibody-dependent monocyte-mediated inhibition of parasite growth in vitro. Infect Immun 66: 11-7. 
